\title{
Dynamic analysis of the semiconductor laser as a current-controlled oscillator in the optical phased-lock loop: applications
}

\author{
Amnon Yariv \\ California Institute of Technology, MS 128-95, 1200 East California Boulevard, Pasadena, California 91125
}

Received February 8, 2005; revised manuscript received May 17, 2005; accepted May 18, 2005

A methodology for treating the semiconductor laser as a current-controlled oscillator in an optical phase-lock loop is presented. The formalism is applied to phase demodulation of optical beams, reduction of phase noise by self-homodyning, and phase locking of a semiconductor laser array. (C) 2005 Optical Society of America OCIS codes: $030.1670,060.1660$.

The voltage-controlled oscillator (VCO) is a key component in radio-frequency networks. Its main applications are in phase-lock loops (PLLs), frequency and phase locking of oscillators, frequency multiplication, and frequency, or phase, demodulation. ${ }^{1}$ An optical analog of a VCO should play a role comparable to that of its radio-frequency counterpart and thus open up new areas of applications involving phasecoherent manipulation of optical signals. A natural candidate for the role of an optical VCO, or rather a current-controlled oscillator, is the semiconductor laser (SCL). ${ }^{2}$ This is due mostly to the sensitivity of the SCL's frequency to injection current and also to its very small response time, shortened by stimulated emission, which promises operation at frequencies exceeding $20 \mathrm{GHz}$.

A schematic of a SCL used in an optical PLL is shown in Fig. 1. The output of the SCL is mixed in a nonlinear (intensity) detector with the optical field of an incoming wave. The output current is amplified and injected into the active region of the SCL. This will cause the response to be limited by the stimulated lifetime, which can extend to frequencies $>20 \mathrm{GHz}$.

The difference between the results presented in this Letter and a number of earlier significant contributions $^{2,3}$ is that here the laser chirp physics whose parameters play a key role in the PLL formalism is explicitly taken into account. Thermal effects, which are structure dependent and, in any case, play a role at relatively low frequencies, are neglected.

Our starting point is the well-known expression that relates the frequency shift of a SCL to the changes in the internal optical energy density ${ }^{4,5}$ :

$$
\Delta \omega_{l}(t)=-\frac{\alpha}{2}\left[\frac{1}{P_{0}} \frac{d(\Delta P)}{d t}+\frac{\epsilon}{\tau_{P}} \Delta P(t)\right] .
$$

Here $\alpha$ is the phase-amplitude coupling factor, $P(t)$ is the photon density, $P_{0}$ is the average photon density, $\epsilon$ is the gain suppression factor, and $\tau_{P}$ is the photon lifetime at transparency of the semiconductor gain medium. Equation (1) is not cast in a form that is very useful for our purposes, since $P(t)$ is not, in practice, a directly controlled physical variable. Such a variable would be $i_{l}(t)$, the injection current to the
SCL. A somewhat lengthy derivation based on the coupled SCL-optical field rate equations ${ }^{4}$ shows that, at frequencies substantially below that of the modulation resonance of the SCL, the photon density is proportional to the excess, i.e., above the threshold, current input to the laser:

$$
P(t)=g\left[i_{l}(t)-i_{l, \mathrm{th}}\right]
$$

where $g=\left(\Gamma_{a} \tau_{p} / e V_{m}\right), V_{m}$ is the mode volume and $\Gamma_{a}$ is the fill factor, approximately the ratio of the active volume to the modal volume and $e$ is the electron charge. We use Eq. (2) to rewrite Eq. (1) as

$$
\begin{aligned}
\Delta \omega_{l}(t) & =-\frac{\alpha}{2}\left[\frac{1}{\left(i_{l 0}-i_{l, \mathrm{th}}\right)} \frac{d\left(\Delta i_{l}\right)}{d t}+\frac{\epsilon g}{\tau_{P}} \Delta i_{l}\right] \\
& =a \frac{d}{d t} \Delta i_{l}+b \Delta i_{l}(t), \\
\omega_{l}(t) & =\omega_{0}+\Delta \omega_{l}(t), \quad \Delta i_{l}(t) \equiv i_{l}(t)-i_{l, \mathrm{th}},
\end{aligned}
$$

where $a=-\alpha / 2\left(i_{l 0}-i_{l, \text { th }}\right), \quad b=-\alpha \epsilon g / 2 \tau_{P}=-\alpha \epsilon \Gamma_{a} / 2 e V_{m}$, and we used $P_{0}=g\left(i_{l 0}-i_{l, \mathrm{th}}\right)$.

Equation (3), the starting point for our analysis, is not valid at frequencies near that of the relaxation resonance of SCL, where the relation between $P$ and $i$ is more complicated. Our model system is illus-

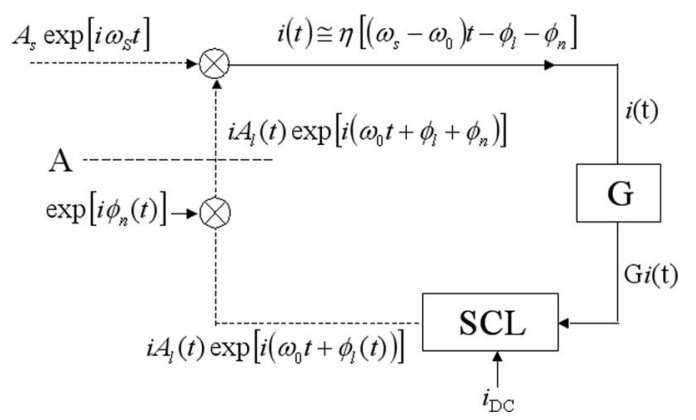

Fig. 1. Model for the laser noise dynamics in an optical PLL. $\phi_{l}(t)$ is a deterministic phase depending explicitly and deterministically on the input (injection) current $G i(t)$. The random phase fluctuations due to the spontaneous emission are represented by $\phi_{n}(t)$. The actual phase of the laser field is $\phi_{l}(t)+\phi_{n}(t)$. 
trated in Fig. 1. The output field of the SCL, $A_{l} \cos \left[\omega_{0} t+\phi_{l}(t)+\pi / 2\right]$, is mixed in a nonlinear optical detector with an incoming field, the signal $A_{s} \cos \left[\omega_{s} t+\phi_{s}(t)\right]$. The resulting current is $i(t)$ $=\eta A_{s} A_{l} \sin \left[\left(\omega_{s}-\omega_{0}\right) t+\phi_{s}(t)-\phi_{l}(t)\right]$, where $\eta$ accounts for the detector conversion of optical power to current. The current is amplified by $G$ and fed to the laser. In this portion we ignore the laser noise so that $\phi_{n}=0$. We assume that the argument $\left[\left(\omega_{s}-\omega_{0}\right) t\right.$ $\left.+\phi_{s}(t)-\phi_{l}(t)\right]$ in the expression for $i(t)$ is sufficiently small that

$$
\begin{gathered}
i(t) \approx K\left[\left(\omega_{s}-\omega_{0}\right) t+\phi_{s}(t)-\phi_{l}(t)\right], \quad K \equiv \eta A_{s} A_{l}, \\
\frac{\mathrm{d} i}{\mathrm{~d} t}=K\left[\left(\omega_{s}-\omega_{0}\right)+\frac{\mathrm{d} \phi_{s}}{\mathrm{~d} t}-\frac{\mathrm{d} \phi_{l}}{\mathrm{~d} t}\right] .
\end{gathered}
$$

Recognizing that $d \phi_{l} / d t=\Delta \omega_{l}(t)$, we use Eq. (3) and the relation $i_{l}=G i$ to rewrite Eq. (5) as

$$
\frac{1}{K} \frac{\mathrm{d}(\Delta i)}{\mathrm{d} t}=\left(\omega_{s}-\omega_{0}\right)+\frac{\mathrm{d} \phi_{s}}{\mathrm{~d} t}-a G \frac{\mathrm{d}}{\mathrm{d} t} \Delta i-b G \Delta i .
$$

Equation (6) is our key result. It relates the current increment $\Delta i$, in the feedback loop, to the phase $\phi_{s}(t)$ of the incoming signal. Consider the static (steady state) behavior of the PLL. At steady state $\mathrm{d} \phi_{s} / \mathrm{d} t$ $=\mathrm{d}(\Delta i) / \mathrm{d} t=0$. The time-independent portion of Eq. (6) is

$$
b G(\Delta i)_{S S}=\omega_{s}-\omega_{0}
$$

From Eq. (3) $\left(\Delta \omega_{l}\right)_{S S}=b G(\Delta i)_{S S}=\omega_{S}-\omega_{0}$ and $\left(\omega_{l}\right)_{S S}$ $=\omega_{0}+\left(\Delta \omega_{l}\right)_{S S}=\omega_{S}$. The application of feedback thus results in an average $(\Delta i)_{S S}$ that is just sufficient to lock the SCL so its average oscillation frequency is identical to signal frequency $\omega_{s}$.

To study the dynamic time-dependent behavior of the system we substitute the value of $(\Delta i)_{S S}$ into Eq. (6). We define $\Delta i(t)=(\Delta i)_{S S}+i_{1}(t)$. The result is the differential equation

$$
\frac{\mathrm{d} i_{1}(t)}{\mathrm{d} t}=K \frac{\mathrm{d} \phi_{s}}{\mathrm{~d} t}-K a G \frac{\mathrm{d} i_{1}(t)}{\mathrm{d} t}-K b G i_{1}
$$

Fourier transformation of this result leads to

$$
\tilde{i}_{1}(\Omega)=\frac{j \Omega K}{K b G+j \Omega(1+K a G)} \widetilde{\phi}_{s}(\Omega),
$$

where $\widetilde{i}_{1}(\Omega)$ and $\widetilde{\phi}_{s}(\Omega)$ are the Fourier transforms of the respective time-varying quantities. Equation (8) is the transfer function relating input-the optical phase $\widetilde{\phi}_{s}(\Omega)$-and the output-electronic current $\tilde{i}_{1}(\Omega)$. According to Eq. (8) the SCL-based PLL can serve as a demodulator for phase-coded optical waves. At low frequencies $\Omega \ll b / a$, Eq. (8) yields

$$
\tilde{i}_{1}(\Omega) \approx \frac{j \Omega}{b G} \widetilde{\phi}_{S}(\Omega), \quad i_{1}(t) \approx \frac{1}{b G} \frac{\mathrm{d}}{\mathrm{d} t} \phi_{S}(t),
$$

and the PLL acts as a frequency demodulator. At high frequencies, $\Omega>b / a$,

$$
\tilde{i}_{1}(\Omega) \approx \frac{K}{1+K a G} \widetilde{\phi}_{s}(\Omega), \quad i_{1}(t) \approx \frac{K}{1+K a G} \phi_{s}(t),
$$

and the input phase information $\phi_{s}(t)$ of the optical signal, in this case, is directly available as a baseband current $i_{1}(t)$ in the feedback loop. The PLL thus acts as a phase demodulator of the incoming (optical) phase-modulated wave, a task for which no simple technical solution exists.

To approximate the magnitude of numbers involved we use the following data in MKS units:

$$
\begin{aligned}
\alpha & =5, \quad i_{0}-i_{\text {th }}=5 \times 10^{-2}, \quad a=50, \quad G=100, \\
K & \equiv \eta A_{l} A_{s} \approx 3 \times 10^{-3}, \quad \epsilon=10^{-23}, \quad \Gamma_{a}=0.1, \\
V & =3 \times 10^{-16}, \\
b & \approx-5 \times 10^{10}, \quad K b G \approx 1.5 \times 10^{10}, \quad K a G \approx 15, \\
a G & \approx 5 \times 10^{3} .
\end{aligned}
$$

Of particular interest is the frequency $b / a$ denoting the transition from essentially frequency demodulation at $\Omega<b / a$ to phase demodulation at $\Omega>b / a$. In our example, $b / 2 \pi a \sim 1.5 \times 10^{8} \mathrm{~Hz}$. Recall that this analysis assumes modulation frequencies $\Omega / 2 \pi$ smaller than the modulation response resonance of the laser, which can be as high as $20 \mathrm{GHz}$. The PLL can thus operate in the frequency region $\Omega / 2 \pi<2$ $\times 10^{10} \mathrm{~Hz}$. A useful relation, to be used below, is that between the input phase $\widetilde{\phi}_{s}(\Omega)$ and the phase $\widetilde{\phi}_{l}(\Omega)$ of the semiconductor laser. From Eq. (3),

$$
\phi_{l}(t)=\int_{0}^{t} \Delta \omega_{l}\left(t^{\prime}\right) \mathrm{d} t^{\prime}=G\left[a i_{1}(t)+b \int_{0}^{t} \Delta i_{1}\left(t^{\prime}\right) \mathrm{d} t^{\prime}\right],
$$

where we neglect a constant phase. A Fourier transformation and Eq. (10) yield

$$
\widetilde{\phi}_{l}(\Omega)=G a \tilde{i}_{1}(\Omega)+\frac{G b}{j \Omega} \widetilde{i}_{1}(\Omega),
$$

$$
\widetilde{\phi}_{l}(\Omega)=\frac{j \Omega+\frac{b}{a}}{\frac{b}{a}+j \Omega\left(1+\frac{1}{K a G}\right)} \widetilde{\phi}_{s}(\Omega) .
$$

In the case $K a G \gg 1, \widetilde{\phi}_{l}(\Omega) \approx \widetilde{\phi}_{s}(\Omega)$ and the laser phase tracks closely, i.e., locks to, that of the signal. We will put this last relation to work next in considering the phase noise of the phase-locked SCL, a point of major theoretical and practical importance. 


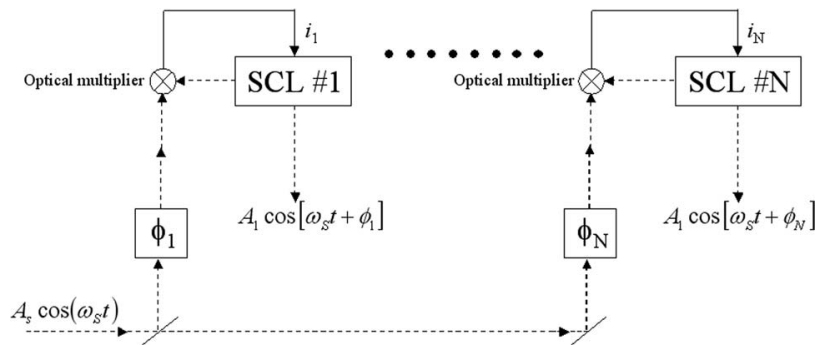

Fig. 2. Individual SCLs all lock to a reference wave at $\omega_{s}$, thus forming a coherent array.

The noise model is illustrated in Fig. 1. It is based on a phasor picture in which each spontaneous emission event perturbs the phase of the otherwise perfectly coherent oscillation represented by a stationary phasor. ${ }^{6}$ The cumulative deviation of the phase due to this noise is $\phi_{n}(t)$. In addition to the random noise $\phi_{n}$ the SCL phase has a deterministic contribution that depends on the input injection current as per Eq. (10). The resulting phase is denoted by $\phi_{1}(t)$. The total laser field is $\phi_{n}(t)+\phi_{1}(t)$.

The noise contribution is obtained in our model by inserting into Fig. 2 a noise multiplier $\exp \left[i \phi_{n}(t)\right]$. We consider the case of an ideal nonmodulated (locking) signal wave so that $\phi_{s}=0$. In analogy with Eq. (4) we have

$$
i(t)=K\left[\left(\omega_{s}-\omega_{0}\right) t-\phi_{l}(t)-\phi_{n}(t)\right] .
$$

Equation (12) is identical to Eq. (4) if, in the latter, we substitute $\phi_{s} \rightarrow-\phi_{n}$. This, combined with Eq. (11), gives

$$
\widetilde{\phi}_{l}(\Omega)=-\frac{j \Omega+\frac{b}{a}}{\frac{b}{a}+j \Omega\left(1+\frac{1}{K a G}\right)} \widetilde{\phi}_{n}(\Omega) .
$$

The actual total phase fluctuation is $\widetilde{\phi}_{\text {laser }}(\Omega)=\widetilde{\phi}_{l}(\Omega)$ $+\widetilde{\phi}_{n}(\Omega)$. In the limit $K a G \gg 1, \widetilde{\phi}_{l}(\Omega)$ and $\widetilde{\phi}_{n}(\Omega)$ are according to Eq. (13) nearly equal in magnitude and of opposite signs. Adding them to obtain the observable phase noise leads to a residual

$$
\widetilde{\phi}_{\text {laser }}(\Omega)=\frac{X\left(\Omega^{2}+\Omega^{2} X+j \Omega \frac{b}{a}\right)}{\Omega^{2}(1+X)^{2}+(b / a)^{2}} \widetilde{\phi}_{n}(\Omega),
$$

where $X=1 / K a G$. In the limit $\Omega \ll b / a, \widetilde{\phi}_{\text {laser }}(\Omega)$ $\rightarrow(j \Omega / K b G) \widetilde{\phi}_{n}(\Omega)$. In the limit $\Omega \gg b / a, \widetilde{\phi}_{\text {laser }}(\Omega)$ $\rightarrow(1 / K a G) \tilde{\phi}_{n}(\Omega)$. In our example $K a G \approx 15, \quad b / a$ $\approx 10^{9}$, so the reduction of the phase-noise spectral density in the PLL can be several orders of magnitude compared to its free-running value. A corresponding narrowing of the laser linewidth follows. Phase noise in the input wave $\phi_{s}$ cannot, obviously, be separated from signal phase modulation and will thus be reflected in the SCL phase.

An interesting and potentially important application of the optical PLL would be that of combining coherently a large number of individual SCLs. The arrangement is shown in Fig. 2. Each laser runs in its own PLL, thus phase locking to the common reference wave at $\omega_{s}$ and consequently to each other. An arbitrary phase shift between the lasers can be introduced either by optical phase shifters in the individual branches leading to each laser or electronically. The implications for steerable laser arrays and adaptive optics are obvious.

This analysis did not include the effects of phase shifts in the electronic feedback loop, which, if substantial, can lead to instabilities and to distortion. In practice, one may anticipate the use of custom electronic integrated circuits, hybrid-integrated with the lasers. To make operation in the important frequency regime of up to $20 \mathrm{GHz}$ possible, one may anticipate the use of fast custom integrated-circuit electronics hybridized with the laser. The laser can be designed and operated at high power densities to push the resonance relaxation frequency up to $30 \mathrm{GHz}$.

In summary, an analysis of optical phase-lock loops based on the use of semiconductor lasers as currentcontrolled oscillators has been presented. The theory predicts wideband phase and frequency locking of the SCL to those of the reference signal wave. Suggested applications include clock recovery, frequency and phase demodulation of frequencies up to $10 \mathrm{GHz}$ and likely higher, and locking and steering of coherent SCL arrays. Major reduction in the phase (noise) spectral density is predicted, which might lead to narrow-line high-coherence linear semiconductor lasers.

The author (ayariv@caltech.edu) acknowledges useful conversations with Steve Pappert (DARPA), Jacob Scheuer (Caltech), and William Marshall (Caltech).

\section{References}

1. See, e.g., J. G. Proakis, Digital Communication, 4th ed. (McGraw-Hill, 2001), pp. 341-357.

2. Y. Yamamoto, O. Nilsson, and S. Saito, IEEE J. Quantum Electron. QE-21, 1919 (1985).

3. M. Ohtsu and S. Kotajima, IEEE J. Quantum Electron. 21, 1905 (1985).

4. T. Koch and J. Bowers, Electron. Lett. 20, 1038 (1984).

5. A. Yariv, Optical Electronics in Modern Communications, 5th ed. (Oxford U. Press, 1997), p. 594.

6. Ref. 5, pp. 393-396. 